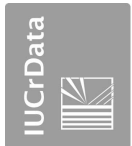

IUCrData

ISSN 2414-3146

Received 28 May 2019

Accepted 17 June 2019

Edited by C. Rizzoli, Universita degli Studi di Parma, Italy

Keywords: crystal structure; nucleobases.

CCDC reference: 1924198

Structural data: full structural data are available from iucrdata.iucr.org

\section{6-Methyluracil: a redetermination of polymorph (II)}

\author{
Gustavo Portalone*
}

Chemistry Department, "Sapienza" University of Rome, P.le A. Moro, 5, I-00185 Rome, Italy. *Correspondence e-mail: gustavo.portalone@uniroma1.it

6-Methyluracil, $\mathrm{C}_{5} \mathrm{H}_{6} \mathrm{~N}_{2} \mathrm{O}_{2}$, exists in two crystalline phases: form (I), monoclinic, space group $P 2{ }_{1} /$ c [Reck et al. (1988). Acta Cryst. A44, 417-421] and form (II), monoclinic, space group C2/c [Leonidov et al. (1993). Russ. J. Phys. Chem. 67, 2220-2223]. The structure of polymorph (II) has been redetermined providing a significant increase in the precision of the derived geometric parameters. In the crystal, molecules form ribbons approximately running parallel to the $c$-axis direction through $\mathrm{N}-\mathrm{H}$.. O hydrogen bonds. The radical differences observed between the crystal packing of the two polymorphs may be responsible in form (II) for an increase in the contribution of the polar canonical forms $\mathrm{C}-$ $\left(\mathrm{O}^{-}\right)=\mathrm{N}-\mathrm{H}^{+}$relative to the neutral canonical form $\mathrm{C}(=\mathrm{O})-\mathrm{N}-\mathrm{H}$ induced by hydrogen-bonding interactions.
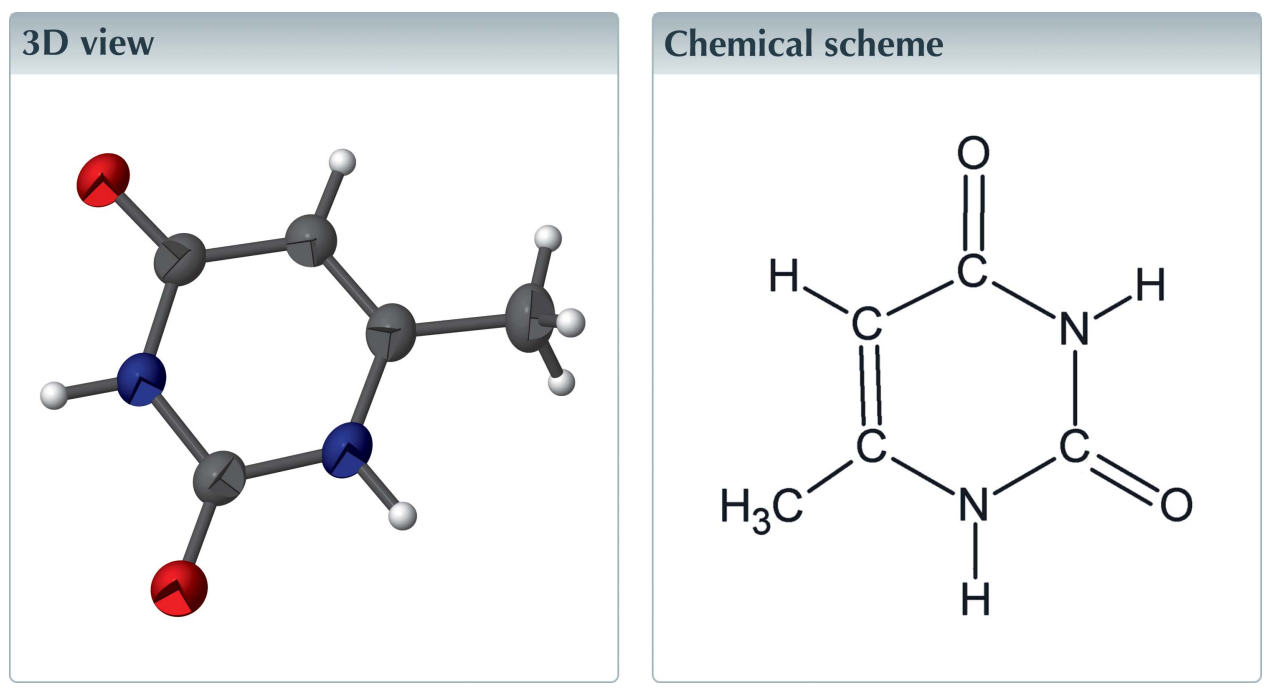

\section{Structure description}

As a result of the fundamental role of nucleic acids in genetic processes of living systems, much attention has been devoted to determining the structure of methylated derivatives of uracil, because methylation of DNA is probably involved in various genetic alterations and in the initiation of carcinogenic processes. In previous theoretical and experimental studies of uracil derivatives from this laboratory (Portalone et al., 1999, 2002; Brunetti et al., 2002; Portalone \& Colapietro, 2004; Portalone, 2010), it has been shown that the hydrogen bonding can change the electronic properties of similar pyrimidine bases experiencing different crystal environments, i.e. showing different sites available for hydrogen-bond interactions.

In this context, 6-methyluracil (6Mura) is particularly attractive, as it exists in two crystalline forms characterized by completely different hydrogen-bonding schemes. A search for crystal structures of the title compound with the Cambridge Structural Database (CSD, version 5.40 updated to May 2019: Groom et al., 2016) gave two hits, as crystalline polymorphs. Form (I), monoclinic space group $P 2_{1} / c$, was determined by 
Table 1

Hydrogen-bond geometry $\left(\AA,^{\circ}\right)$.

\begin{tabular}{lllll}
\hline$D-\mathrm{H} \cdots A$ & $D-\mathrm{H}$ & $\mathrm{H} \cdots A$ & $D \cdots A$ & $D-\mathrm{H} \cdots A$ \\
\hline $\mathrm{N} 1-\mathrm{H} 1 \cdots \mathrm{O} 1^{\mathrm{i}}$ & $0.91(2)$ & $1.95(2)$ & $2.8594(17)$ & $174.0(16)$ \\
$\mathrm{N} 3-\mathrm{H} 3 \cdots \mathrm{O} 2^{\mathrm{ii}}$ & $0.93(2)$ & $1.90(2)$ & $2.8246(18)$ & $171.5(18)$ \\
\hline
\end{tabular}

Symmetry codes: (i) $-x,-y+1,-z$; (ii) $-x+\frac{1}{2},-y+\frac{1}{2},-z$.

XRPD at $R=0.040$ (Reck et al., 1988). Form (II), monoclinic space group $C 2 / c$, was determined by RT single-crystal diffraction performed on a Syntex P1 diffractometer at $R=$ 0.085 (Leonidov et al., 1993). Interestingly, Leonidov and coworkers analysed the differences in the molecular geometries of 6Mura in form (I) and (II), and came to the conclusion that the observed discrepancies in the corresponding bond distances and bond angles were insignificant. Therefore, to verify this assumption, it was considered worthwhile and of significant chemical interest to redetermine the crystal structure of form (II) to a better precision. The present determination $(R=0.048)$, although based on RT data collection, decreases the s.u.s on the bond distances and bond angles to about one third to one fourth of those in the original determination.

In the asymmetric unit, 6Mura is present as the diketo tautomer (Fig. 1). As previously mentioned, the crystal structures of the two polymorphs are radically different. In form (II), infinite ribbons approximately running parallel to the $c$-axis direction are connected by $\mathrm{N}-\mathrm{H} \cdots \mathrm{O}$ hydrogen bonds (Fig. 2). Within a ribbon, each molecule is linked to two adjacent molecules via centrosymmetric, pairwise N1$\mathrm{H} 1 \cdots \mathrm{O} 1$ and $\mathrm{N} 3-\mathrm{H} 3 \cdots \mathrm{O} 2$ hydrogen bonds (Table 1). In

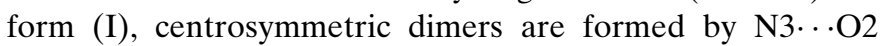
hydrogen bonds. These dimers are then connected through $\mathrm{N} 1 \cdots \mathrm{O} 2$ hydrogen bonds to form layers approximately parallel to the $b c$ plane, leaving the $\mathrm{O} 1$ atom free from hydrogen-bonding interactions (Fig. 3).

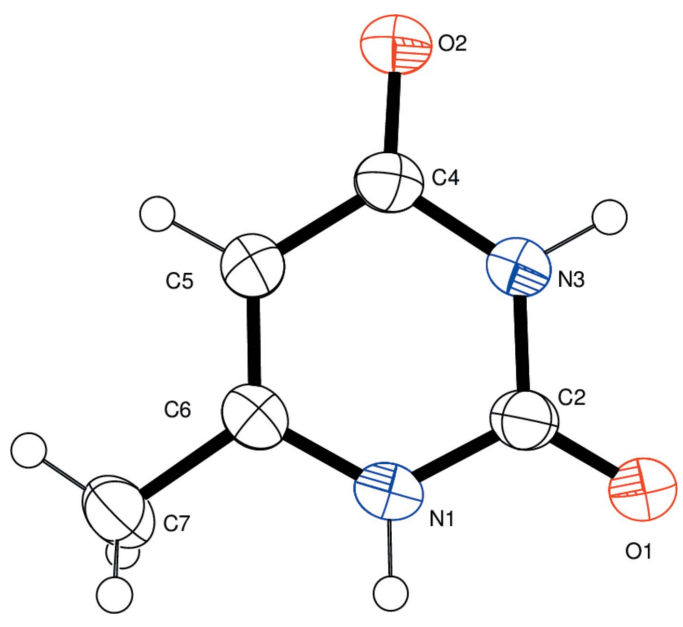

Figure 1

The asymmetric unit of 6Mura in form (II), showing the atom-labelling scheme. Displacements ellipsoids are at the $50 \%$ probability level. $\mathrm{H}$ atoms are shown as small spheres of arbitrary radii.

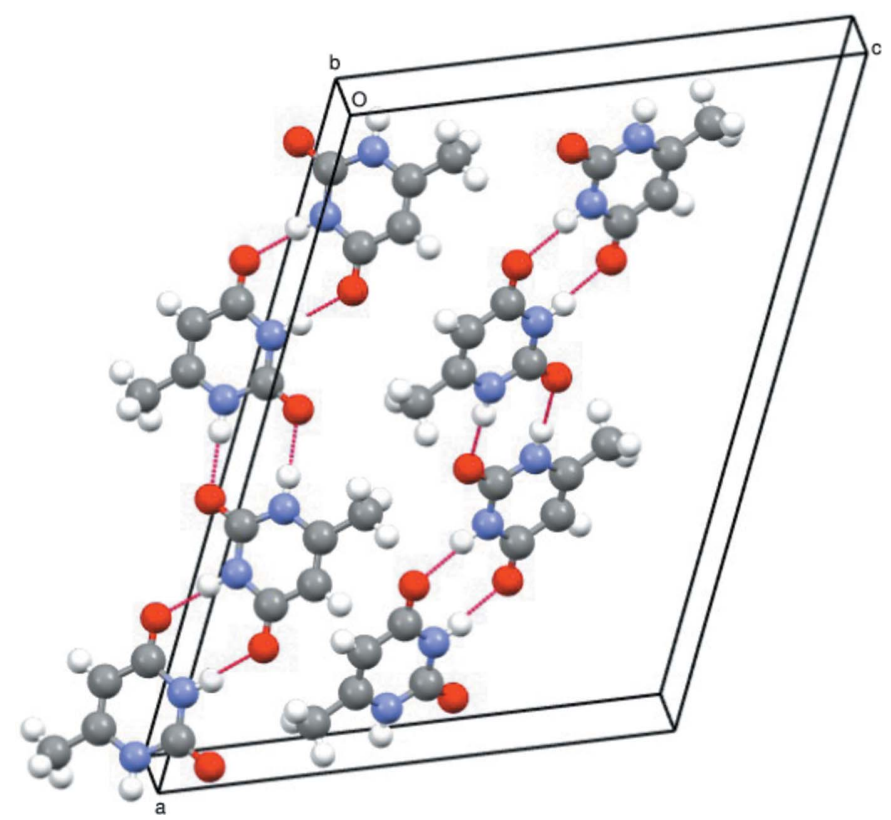

Figure 2

Crystal packing diagram for 6Mura in form (II), viewed approximately down $b$. All atoms are shown as small spheres of arbitrary radii. Hydrogen bonding is indicated by red dashed lines.

The effect of the different crystal environments on the molecular geometry of the two polymorphs can be appreciated in particular in the region involved in the hydrogen bonding, and mainly consists in a concerted distortion of the $\mathrm{C}=\mathrm{O}$ and $\mathrm{C}-\mathrm{N}$ bond distances. Indeed, by comparing the molecular

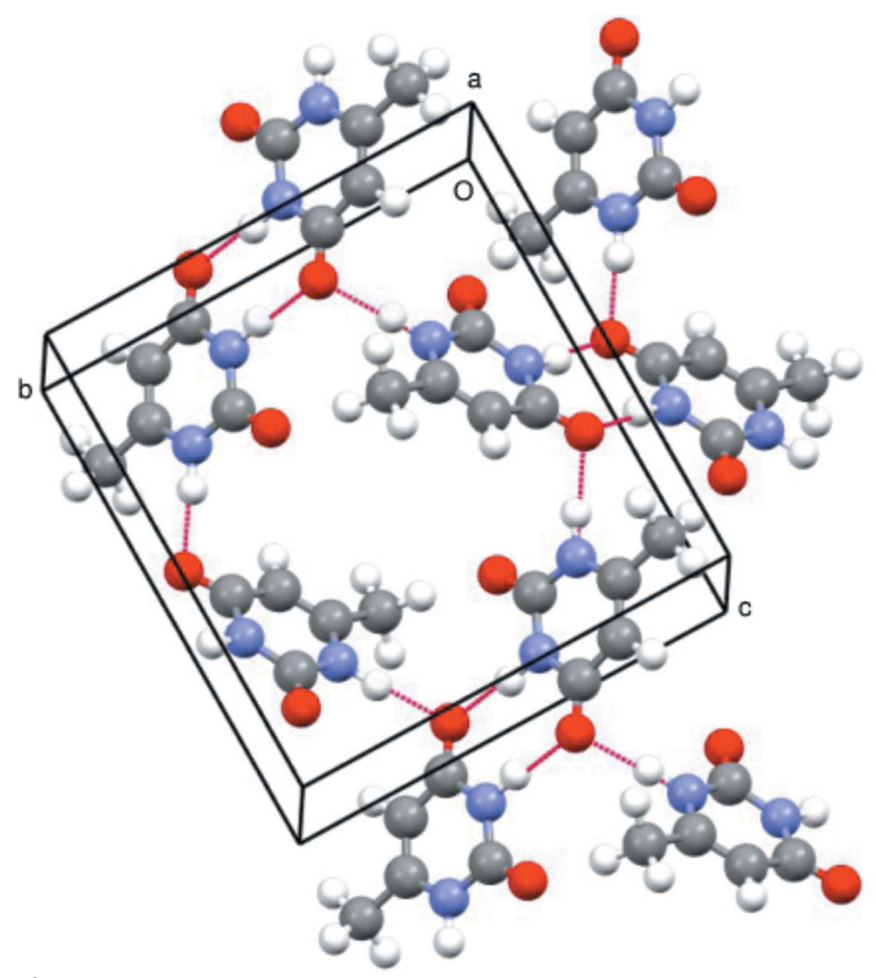

Figure 3

Crystal packing diagram for 6Mura in form (I), viewed approximately down $a$. All atoms are shown as small spheres of arbitrary radii. Hydrogen bonding is indicated by red dashed lines. 
<smiles></smiles>

(la)<smiles>Cc1cc([O-])[nH+]c(=O)[nH]1</smiles>

(lb)
Figure 4

Polar canonical forms of 6Mura in form (II).

geometry of the almost planar $\mathrm{O} 1=\mathrm{C} 2-\mathrm{N} 3-\mathrm{C} 4=\mathrm{O} 2$ fragment in the two polymorphs, in passing from form (I) to form (II):

(i) the two carbonyl bond distances are longer by $0.008-$ 0.035 (2) $\AA$;

(ii) the $\mathrm{N} 3-\mathrm{C} 2$ and $\mathrm{N} 3-\mathrm{C} 4$ bond distances are shorter by $0.031-0.035$ (2) ̊.

Therefore, the observed structural changes in form (II) can be interpreted, in terms of $\mathrm{VB}$ theory, as an increase in the contribution of the polar canonical forms $\mathrm{C}-\left(\mathrm{O}^{-}\right)=\mathrm{N}^{+}-\mathrm{H}$ [(Ia) and (Ib) in Fig. 4], which are better proton donors and acceptors than the neutral canonical form $\mathrm{C}(=\mathrm{O})-\mathrm{N}-\mathrm{H}$ to form hydrogen-bonding interactions.

\section{Synthesis and crystallization}

6-Methyluracil (Sigma Aldrich, 97\%) was subjected to further purification by successive sublimation under reduced pressure. $1 \mathrm{mmol}$ was dissolved in DMF $(3 \mathrm{ml})$ and stirred for $8 \mathrm{~h}$ at $50^{\circ} \mathrm{C}$. The solution was then cooled to room temperature and allowed to slowly evaporate to give, after two weeks, colourless crystals of polymorph (II) suitable for X-ray analysis. Several trials of data collection at LT using a crystal of polymorph (II) mounted under paraffin oil in a nylon loop failed, as the sample cracked when slowly cooled in liquid nitrogen.

\section{Refinement}

Crystal data, data collection and structure refinement details are summarized in Table 2.

\section{References}

Agilent (2014). CrysAlis PRO. Agilent Technologies Ltd, Yarnton, England.
Table 2

Experimental details.

\begin{tabular}{|c|c|}
\hline \multicolumn{2}{|l|}{ Crystal data } \\
\hline Chemical formula & $\mathrm{C}_{5} \mathrm{H}_{6} \mathrm{~N}_{2} \mathrm{O}_{2}$ \\
\hline$M_{\mathrm{r}}$ & 126.12 \\
\hline Crystal system, space group & Monoclinic, $C 2 / c$ \\
\hline Temperature (K) & 298 \\
\hline$a, b, c(\AA)$ & $20.572(3), 3.9052(5), 14.811(3)$ \\
\hline$\beta\left({ }^{\circ}\right)$ & $110.95(2)$ \\
\hline$V\left(\AA^{3}\right)$ & $1111.2(4)$ \\
\hline$Z$ & 8 \\
\hline Radiation type & Мo $K \alpha$ \\
\hline$\mu\left(\mathrm{mm}^{-1}\right)$ & 0.12 \\
\hline Crystal size $(\mathrm{mm})$ & $0.14 \times 0.11 \times 0.08$ \\
\hline \multicolumn{2}{|l|}{ Data collection } \\
\hline Diffractometer & Agilent Xcalibur Sapphire3 \\
\hline Absorption correction & $\begin{array}{l}\text { Multi-scan (CrysAlis PRO; } \\
\text { Agilent, 2014) }\end{array}$ \\
\hline$T_{\min }, T_{\max }$ & $0.770,1.000$ \\
\hline $\begin{array}{l}\text { No. of measured, independent and } \\
\text { observed }[I>2 \sigma(I)] \text { reflections }\end{array}$ & $8110,1400,1154$ \\
\hline$R_{\text {int }}$ & 0.044 \\
\hline$(\sin \theta / \lambda)_{\max }\left(\AA^{-1}\right)$ & 0.671 \\
\hline \multicolumn{2}{|l|}{ Refinement } \\
\hline$R\left[F^{2}>2 \sigma\left(F^{2}\right)\right], w R\left(F^{2}\right), S$ & $0.048,0.133,1.11$ \\
\hline No. of reflections & 1400 \\
\hline No. of parameters & 91 \\
\hline $\mathrm{H}$-atom treatment & $\begin{array}{l}\mathrm{H} \text { atoms treated by a mixture of } \\
\text { independent and constrained } \\
\text { refinement }\end{array}$ \\
\hline$\Delta \rho_{\max }, \Delta \rho_{\min }\left(\mathrm{e} \AA^{-3}\right)$ & $0.27,-0.17$ \\
\hline
\end{tabular}

Computer programs: CrysAlis PRO (Agilent, 2014), SIR2004 (Burla et al., 2005), SHELXL2014 (Sheldrick, 2015) and WinGX (Farrugia, 2012).

Brunetti, B., Portalone, G. \& Piacente, V. (2002). J. Chem. Eng. Data, 47, 17-19.

Burla, M. C., Caliandro, R., Camalli, M., Carrozzini, B., Cascarano, G. L., De Caro, L., Giacovazzo, C., Polidori, G. \& Spagna, R. (2005). J. Appl. Cryst. 38, 381-388.

Farrugia, L. J. (2012). J. Appl. Cryst. 45, 849-854.

Groom, C. R., Bruno, I. J., Lightfoot, M. P. \& Ward, S. C. (2016). Acta Cryst. B72, 171-179.

Leonidov, N. B., Zorkij, P. M., Masunov, A. E., Gladkikh, O. P., Bel'skii, V. K., Dzyabchenko, A. V. \& Ivanov, S. A. (1993). Russ. J. Phys. Chem. 67, 2220-2223.

Portalone, G. (2010). Acta Cryst. C66, o295-o301.

Portalone, G., Ballirano, P. \& Maras, A. (2002). J. Mol. Struct. 608, $35-$ 39.

Portalone, G., Bencivenni, L., Colapietro, M., Pieretti, A. \& Ramondo, F. (1999). Acta Chem. Scand. 53, 57-68.

Portalone, G. \& Colapietro, M. (2004). J. Chem. Crystallogr. 34, 609612.

Reck, G., Kretschmer, R.-G., Kutschabsky, L. \& Pritzkow, W. (1988). Acta Cryst. A44, 417-421.

Sheldrick, G. M. (2015). Acta Cryst. C71, 3-8. 


\section{full crystallographic data}

IUCrData (2019). 4, x190861 [https://doi.org/10.1107/S2414314619008617]

6-Methyluracil: a redetermination of polymorph (II)

\section{Gustavo Portalone}

6-Methyluracil

Crystal data

$\mathrm{C}_{5} \mathrm{H}_{6} \mathrm{~N}_{2} \mathrm{O}_{2}$

$M_{r}=126.12$

Monoclinic, $C 2 / c$

$a=20.572(3) \AA$

$b=3.9052(5) \AA$

$c=14.811(3) \AA$

$\beta=110.95(2)^{\circ}$

$V=1111.2(4) \AA^{3}$

$Z=8$

$F(000)=528$

$D_{\mathrm{x}}=1.508 \mathrm{Mg} \mathrm{m}^{-3}$

Mo $K \alpha$ radiation, $\lambda=0.710689 \AA$

Cell parameters from 2315 reflections

$\theta=2.9-30.6^{\circ}$

$\mu=0.12 \mathrm{~mm}^{-1}$

$T=298 \mathrm{~K}$

Tablets, colourless

$0.14 \times 0.11 \times 0.08 \mathrm{~mm}$

\section{Data collection}

Agilent Xcalibur Sapphire3 diffractometer

Radiation source: fine-focus sealed X-ray tube, Enhance (Mo) X-ray Source

Graphite monochromator

Detector resolution: 16.0696 pixels $\mathrm{mm}^{-1}$

$\omega$ and $\varphi$ scans

Absorption correction: multi-scan

(CrysAlis PRO; Agilent, 2014)

$T_{\min }=0.770, T_{\max }=1.000$

8110 measured reflections

1400 independent reflections

1154 reflections with $I>2 \sigma(I)$

$R_{\text {int }}=0.044$

$\theta_{\max }=28.5^{\circ}, \theta_{\min }=3.0^{\circ}$

$h=-27 \rightarrow 27$

$k=-5 \rightarrow 5$

$l=-19 \rightarrow 19$

\section{Refinement}

Refinement on $F^{2}$

Least-squares matrix: full

$R\left[F^{2}>2 \sigma\left(F^{2}\right)\right]=0.048$

$w R\left(F^{2}\right)=0.133$

$S=1.11$

1400 reflections

91 parameters

0 restraints

Primary atom site location: structure-invariant direct methods

Secondary atom site location: difference Fourier map

Hydrogen site location: mixed

$\mathrm{H}$ atoms treated by a mixture of independent and constrained refinement

$w=1 /\left[\sigma^{2}\left(F_{\mathrm{o}}^{2}\right)+(0.0609 P)^{2}+0.4161 P\right]$ where $P=\left(F_{\mathrm{o}}^{2}+2 F_{\mathrm{c}}^{2}\right) / 3$

$(\Delta / \sigma)_{\max }<0.001$

$\Delta \rho_{\max }=0.27 \mathrm{e} \AA^{-3}$

$\Delta \rho_{\min }=-0.17$ e $\AA^{-3}$

\section{Special details}

Geometry. All esds (except the esd in the dihedral angle between two 1.s. planes) are estimated using the full covariance matrix. The cell esds are taken into account individually in the estimation of esds in distances, angles and torsion angles; correlations between esds in cell parameters are only used when they are defined by crystal symmetry. An approximate (isotropic) treatment of cell esds is used for estimating esds involving 1.s. planes. 
Refinement. The N-bound $\mathrm{H}$ atoms were located in a difference Fourier map and refined freely. All other $\mathrm{H}$ atoms were placed geometrically and refined using a riding atom approximation, with $\mathrm{C}-\mathrm{H}=0.97 \AA$, and with $U_{\text {iso }}(\mathrm{H})=1.2 U_{\text {eq }}(\mathrm{C})$ or $1.5 U_{\mathrm{eq}}(\mathrm{C})$ for methyl $\mathrm{H}$ atoms. A rotating model was used for the methyl groups.

Fractional atomic coordinates and isotropic or equivalent isotropic displacement parameters $\left(\AA^{2}\right)$

\begin{tabular}{lllll}
\hline & $x$ & $y$ & $z$ & $U_{\text {iso }} * / U_{\text {eq }}$ \\
\hline O1 & $0.06097(6)$ & $0.5147(3)$ & $-0.06247(9)$ & $0.0486(4)$ \\
O2 & $0.26885(6)$ & $0.0108(3)$ & $0.10454(9)$ & $0.0468(3)$ \\
N1 & $0.07366(7)$ & $0.2401(3)$ & $0.07840(9)$ & $0.0380(3)$ \\
H1 & $0.0293(10)$ & $0.304(5)$ & $0.0715(13)$ & $0.049(5)^{*}$ \\
C2 & $0.09750(8)$ & $0.3466(4)$ & $0.00759(11)$ & $0.0366(4)$ \\
N3 & $0.16426(6)$ & $0.2545(3)$ & $0.02038(9)$ & $0.0361(3)$ \\
H3 & $0.1820(10)$ & $0.333(5)$ & $-0.0258(15)$ & $0.057(5)^{*}$ \\
C4 & $0.20846(7)$ & $0.0710(4)$ & $0.09840(11)$ & $0.0357(4)$ \\
C5 & $0.17907(7)$ & $-0.0298(4)$ & $0.16844(11)$ & $0.0372(4)$ \\
H5 & 0.2066 & -0.1641 & 0.2240 & $0.045^{*}$ \\
C6 & $0.11357(8)$ & $0.0595(4)$ & $0.15811(11)$ & $0.0359(4)$ \\
C7 & $0.08038(9)$ & $-0.0227(5)$ & $0.23014(13)$ & $0.0488(5)$ \\
H7A & 0.1100 & -0.1790 & 0.2783 & $0.073^{*}$ \\
H7B & 0.0354 & -0.1289 & 0.1975 & $0.073^{*}$ \\
H7C & 0.0742 & 0.1864 & 0.2615 & $0.073^{*}$ \\
\end{tabular}

Atomic displacement parameters $\left(\hat{A}^{2}\right)$

\begin{tabular}{lllllll}
\hline & $U^{11}$ & $U^{22}$ & $U^{33}$ & $U^{12}$ & $U^{13}$ & $U^{23}$ \\
\hline O1 & $0.0372(6)$ & $0.0662(8)$ & $0.0451(7)$ & $0.0128(5)$ & $0.0178(5)$ & $0.0152(5)$ \\
O2 & $0.0319(6)$ & $0.0639(8)$ & $0.0475(7)$ & $0.0091(5)$ & $0.0178(5)$ & $0.0105(5)$ \\
N1 & $0.0300(7)$ & $0.0451(7)$ & $0.0423(7)$ & $0.0023(5)$ & $0.0171(5)$ & $0.0025(6)$ \\
C2 & $0.0312(7)$ & $0.0414(8)$ & $0.0387(8)$ & $0.0008(6)$ & $0.0142(6)$ & $-0.0007(6)$ \\
N3 & $0.0308(6)$ & $0.0435(7)$ & $0.0365(7)$ & $0.0021(5)$ & $0.0150(5)$ & $0.0031(5)$ \\
C4 & $0.0304(7)$ & $0.0389(7)$ & $0.0383(8)$ & $-0.0006(6)$ & $0.0130(6)$ & $-0.0022(6)$ \\
C5 & $0.0344(8)$ & $0.0416(8)$ & $0.0359(8)$ & $0.0005(6)$ & $0.0129(6)$ & $0.0038(6)$ \\
C6 & $0.0369(8)$ & $0.0356(7)$ & $0.0385(8)$ & $-0.0035(6)$ & $0.0174(6)$ & $-0.0016(6)$ \\
C7 & $0.0496(10)$ & $0.0552(10)$ & $0.0519(11)$ & $-0.0006(7)$ & $0.0308(8)$ & $0.0042(7)$ \\
\hline
\end{tabular}

Geometric parameters $\left(\AA,{ }^{\circ}\right)$

\begin{tabular}{llll}
\hline $\mathrm{O} 1-\mathrm{C} 2$ & $1.2308(19)$ & $\mathrm{C} 4-\mathrm{C} 5$ & $1.430(2)$ \\
$\mathrm{O} 2-\mathrm{C} 4$ & $1.2352(18)$ & $\mathrm{C} 5-\mathrm{C} 6$ & $1.347(2)$ \\
$\mathrm{N} 1-\mathrm{C} 6$ & $1.368(2)$ & $\mathrm{C} 5-\mathrm{H} 5$ & 0.9700 \\
$\mathrm{~N} 1-\mathrm{C} 2$ & $1.372(2)$ & $\mathrm{C} 6-\mathrm{C} 7$ & $1.493(2)$ \\
$\mathrm{N} 1-\mathrm{H} 1$ & $0.91(2)$ & $\mathrm{C} 7-\mathrm{H} 7 \mathrm{~A}$ & 0.9701 \\
$\mathrm{C} 2-\mathrm{N} 3$ & $1.3657(19)$ & $\mathrm{C} 7-\mathrm{H} 7 \mathrm{~B}$ & 0.9701 \\
$\mathrm{~N} 3-\mathrm{C} 4$ & $1.3872(19)$ & $\mathrm{C} 7-\mathrm{H} 7 \mathrm{C}$ & 0.9701 \\
$\mathrm{~N} 3-\mathrm{H} 3$ & $0.93(2)$ & & \\
$\mathrm{C} 6-\mathrm{N} 1-\mathrm{C} 2$ & $123.21(13)$ & $\mathrm{C} 6-\mathrm{C} 5-\mathrm{C} 4$ & $120.79(14)$
\end{tabular}




$\begin{array}{llll}\mathrm{C} 6-\mathrm{N} 1-\mathrm{H} 1 & 120.2(12) & \mathrm{C} 6-\mathrm{C} 5-\mathrm{H} 5 & 119.6 \\ \mathrm{C} 2-\mathrm{N} 1-\mathrm{H} 1 & 116.6(12) & \mathrm{C} 5-\mathrm{C} 6-\mathrm{N} 1 & 119.92(14) \\ \mathrm{O} 1-\mathrm{C} 2-\mathrm{N} 3 & 122.54(14) & \mathrm{C} 5-\mathrm{C} 6-\mathrm{C} 7 & 123.91(15) \\ \mathrm{O} 1-\mathrm{C} 2-\mathrm{N} 1 & 121.93(14) & \mathrm{N} 1-\mathrm{C} 6-\mathrm{C} 7 & 116.16(13) \\ \mathrm{N} 3-\mathrm{C} 2-\mathrm{N} 1 & 115.53(14) & \mathrm{C} 6-\mathrm{C} 7-\mathrm{H} 7 \mathrm{~A} & 109.5 \\ \mathrm{C} 2-\mathrm{N} 3-\mathrm{C} 4 & 125.36(13) & \mathrm{C} 6-\mathrm{C} 7-\mathrm{H} 7 \mathrm{~B} & 109.5 \\ \mathrm{C} 2-\mathrm{N} 3-\mathrm{H} 3 & 116.6(12) & \mathrm{H} 7 \mathrm{~A}-\mathrm{C} 7-\mathrm{H} 7 \mathrm{~B} & 109.5 \\ \mathrm{C} 4-\mathrm{N} 3-\mathrm{H} 3 & 117.9(12) & \mathrm{C} 6-\mathrm{C} 7-\mathrm{H} 7 \mathrm{C} & 109.5 \\ \mathrm{O} 2-\mathrm{C} 4-\mathrm{N} 3 & 120.14(14) & \mathrm{H} 7 \mathrm{~A}-\mathrm{C} 7-\mathrm{H} 7 \mathrm{C} & 109.5 \\ \mathrm{O} 2-\mathrm{C} 4-\mathrm{C} 5 & 124.69(14) & \mathrm{H} 7 \mathrm{~B}-\mathrm{C} 7-\mathrm{H} 7 \mathrm{C} & \\ \mathrm{N} 3-\mathrm{C} 4-\mathrm{C} 5 & 115.16(13) & & 177.08(14) \\ & & & -1.6(2) \\ \mathrm{C} 6-\mathrm{N} 1-\mathrm{C} 2-\mathrm{O} 1 & -178.53(14) & \mathrm{O} 2-\mathrm{C} 4-\mathrm{C} 5-\mathrm{C} 6 & 1.9(2) \\ \mathrm{C} 6-\mathrm{N} 1-\mathrm{C} 2-\mathrm{N} 3 & 1.3(2) & \mathrm{N} 3-\mathrm{C} 4-\mathrm{C} 5-\mathrm{C} 6 & -177.22(14) \\ \mathrm{O} 1-\mathrm{C} 2-\mathrm{N} 3-\mathrm{C} 4 & 178.78(14) & \mathrm{C} 4-\mathrm{C} 5-\mathrm{C} 6-\mathrm{N} 1 & -1.8(2) \\ \mathrm{N} 1-\mathrm{C} 2-\mathrm{N} 3-\mathrm{C} 4 & -1.0(2) & \mathrm{C} 4-\mathrm{C} 5-\mathrm{C} 6-\mathrm{C} 7 & 177.42(14) \\ \mathrm{C} 2-\mathrm{N} 3-\mathrm{C} 4-\mathrm{O} 2 & -177.57(14) & \mathrm{C} 2-\mathrm{N} 1-\mathrm{C} 6-\mathrm{C} 5 & \\ \mathrm{C} 2-\mathrm{N} 3-\mathrm{C} 4-\mathrm{C} 5 & 1.2(2) & \mathrm{C} 2-\mathrm{N} 1-\mathrm{C} 6-\mathrm{C} 7 & \\ & & & \end{array}$

Hydrogen-bond geometry $\left(A,{ }^{\circ}\right)$

\begin{tabular}{lllll}
\hline$D-\mathrm{H} \cdots A$ & $D-\mathrm{H}$ & $\mathrm{H} \cdots A$ & $D \cdots A$ & $D-\mathrm{H} \cdots A$ \\
\hline $\mathrm{N} 1-\mathrm{H} 1 \cdots \mathrm{O} 1^{\mathrm{i}}$ & $0.91(2)$ & $1.95(2)$ & $2.8594(17)$ & $174.0(16)$ \\
$\mathrm{N} 3-\mathrm{H} 3 \cdots \mathrm{O} 2^{\mathrm{ii}}$ & $0.93(2)$ & $1.90(2)$ & $2.8246(18)$ & $171.5(18)$ \\
\hline
\end{tabular}

Symmetry codes: (i) $-x,-y+1,-z$; (ii) $-x+1 / 2,-y+1 / 2,-z$. 